\title{
The Mechanism of Crowd Stampede Based on Case Statistics through SNA Method
}

\author{
Xinyao GUO*, Linqing NIU, Hongyan GUAN
}

\begin{abstract}
Stampede is a concern of urban pubic security management. The current academic research focus is the identification of risk factors of trampling accidents and determination of correlation patterns and accident-causing mechanisms among stampede elements in order to effectively obtain the influencing factors of stampede and clarify the transmission routes of stampede risk factors. Previous index cases were scrutinized and analyzed in 78 typical stampedes from 2010 - 2019 based on "pedestrianequipment-environment-management" framework, and 17 influencing factors of stampede by adopting a conceptual coding method were obscured. Then, the degree centrality, intermediate centrality and respective weights of the influencing factors were calculated based on the social network analysis (SNA) method. The influencing level of the factors was signified, and the transmission mechanism of risk in the system network was determined. The results reveal that the degree centrality and weight with conspicuous features of over-density of crowds, pedestrian swarming and falling, and insufficient on-site transactions contribute the most. This finding indicates that these factors play a relatively major role in the stampede system. Furthermore, the intermediate centrality of insufficient on-site transactions is the top factor, meaning that this factor has a strong controlling force in the incident system and considerably influences other factors. This study shows that the SNA method is feasible in analyzing the mechanism of stampede incidents, simultaneously addressing the shortcomings of the linear statistical model of factors and providing theoretical support for comprehensive control of crowd risk.
\end{abstract}

Keywords: accident mechanism; risk analysis; social network analysis; Stampede

\section{INTRODUCTION}

The advancement of global urbanization has made crowd gathering common [1]. Many various types of mass emergencies due to pressing crowds have occurred worldwide in recent years, such as the 2014 Shanghai Stampede, the 2015 Saudi Mecca Hajj, and the 2019 Karbala Stampede in Iraq. Cities, as a special public security carrier, are surrounded by increasing risks and threats [2]. Crowd stampede, characterized by suddenness and negligibility, often happens during conglomeration events or in crowded places, such as subway stations and gymnasiums. Stampede is considered a human-made disaster that is not only subject to emergencies, meteorological conditions, geographical environment, and pedestrian psychology but also correlated to on-site crowd monitoring and management measures, including factors such as intricate relationships [3, 4]. The efforts of scientifically identifying the influencing factors of stampede, delving into the interaction mechanism between differentiated factors, and illustrating the triggering mechanism of crowd stampede are organizational premises to prevent controllable accidents.

Previous research on influencing factors of stampede principally concentrates on statistics of accident types, emergencies, crowd panic, pedestrian falling, and other behaviour errors of accident subjects, and less attention has been paid to organizational factors that lead to these behavioural errors, namely, on-site transactions, crowd monitoring, and emergency plans [5-8]. Research on the triggering mechanisms of stampede accidents mostly uses computer modelling, simulation, and subjective analysis to introduce the time logic and evolution process of stampede into incident analysis and discusses the effect of humanmade errors on the triggering of trampling incidents from the micro level $[9,10]$. Existing research on crowd stampede generally prioritizes analysis of crowd-level behaviour errors in risk events and is insufficient in discussion on organizational-level management factors. Insufficient attention has been paid to the interaction between risk factors, particularly the lack of in-depth research on the causality and mechanism of risk factors $[11,12]$. This study screens out the risk factors of accidents from multiple perspectives such as pedestrian, equipment, environment and management, analyzes the network correlation mode and effectiveness between different influencing factors, determines the path of risk transmission in the system, and explores the mechanism of accident occurrence, providing practical guiding significance for the reduction of stampede risk.

\section{STATE OF THE ART}

In terms of the identification and screening of risk factors for crowd stampede incidents, most existing literature analyzed the correlation between factors to determine the influencing range of each factor on stampede accidents by adopting a mathematical statistics method $[13,14]$. Wang et al. used soft system methodology to carry out incident deduction and analysis on the Rainbow Bridge stampede in Miyun, China in 2004, concluding that the main causes of the trampling accident are the insufficient prediction of the number of tourists by management, communication barriers, and design errors of the bridge [15]. Liu et al. established a data envelopment analysis (DEA) model for the risk assessment of stampede by collecting information on 13 metro stations in Beijing, China to determine risk input and output vectors of crowd stampede in metro stations [16]. Based on data of religious, entertainment, and political rallies in India over the past 50 years, Faisel contended that stampede is considered the main danger that may occur in large-scale assembly activities in India. He argued that a simple incident, deliberate behaviour, and even a rumor can cause crowd riots [17]. Alaska et al. comprehensively summarized and analyzed accidents through public Mecca pilgrimage data on crowd stampede with the support of Medline and OVID, and they proposed the most appropriate way to arrange pilgrims to avoid crowd trampling accidents [18].

In general, current studies on the causes and evolution mechanism of stampede are reified into two stereotypes: case analysis and general summary analysis types. They 
are manifested as time evolution [19], subjectivity [20], emotional contagion, and abnormal behaviour blindness [21]. Helbing et al. considered that the domino effect of pedestrians falling due to crowd churning is the crucial risk factor for stampede after exposing the occurrence process of stampede accidents according to qualitative analysis of crowd stampede case data [22]. Ramesh found that the main cause of crowd stampedes deteriorated from crowding and swarming results from the intensifying pressure of crowds caused by the presence of obstacles in the crowds. In other words, panic escape leads to competitive crowding of crowds to achieve a goal [23]. By contrast, Shiwakoti's research on the behaviour of emergency evacuation of 1,134 subway passengers found that in emergencies, passengers prefer to abide by the rules than to move out, care for each other than compete with others, and pursue reliable dependent paths than different approaches [24]. Zhao determined the relative issues of crowd evacuation under the circumstance of panic triggered by obstacles by setting up obstructions near the exit [25]. Dias expedited citizen's escape behaviour in complex situations from a behavioural perspective. Her results suggest that higher turning angles can reduce the speed of pedestrians, further significantly decreasing the crowds. Nevertheless, intersections and merged roads will spell the stalling and moving of crowds, which easily cause crowd trampling and rolling [26].

In summary, current research on crowd stampedes focuses on inducements, such as pedestrian behaviour, psychology, and crowd status, which directly lead to the occurrence of accidents, but the research lacks profound recognition and analysis of management factors and the failure of security facilities. Moreover, in terms of the trigger mechanism and evolution characteristics of stampedes, the research uses the angles of mathematical statistics, behaviour simulation, and spatio-temporal state evolution. The research focuses less on literal records on the analysis of the relationship between factors, the behaviour mechanism and intensity of risk factors, and the induced evolution process of stampede.

Therefore, to compensate for the shortcomings of the existing research, this study uses a "Pedestrian-EquipmentEnvironment-Management" model to identify and define the influencing factors of stampedes with the support of case analysis and questionnaires, establishes an influencing matrix among factors by the social network analysis (SNA) method, measures the intensity effect of each influencing factor through the degree centrality, and verifies the accuracy of the SNA method via the weight analysis results of historical cases. Such an effective mechanism to comprehensively analyze stampede accidents will be conductive to clarifying the focus of risk management for managers.

The remainder of this study is arranged as follows. Section 3 illustrates the screening and extraction method of the influencing factors of stampede on runways, the influencing matrix constructed by SNA, the calculation of centrality, the gray correlation model construction method, and the weight coefficient expression method. Section 4 provides the corresponding result analysis and relative discussion, pointing out the effect mechanism of each stampede risk factor. Section 5 draws conclusions.

\section{METHODOLOGY \\ 3.1 Influencing Factor Analysis Framework and Conceptual Coding}

The identification of influencing factors underpins the analysis of influencing mechanisms [27]. The influencing factors of crowd stampede accidents are grouped into four perspectives: pedestrian, equipment, environment, and management, which are entwined with pedestrian movement and psychological state, crowd monitoring and controlling equipment, dense crowd scene environment, and organizational security management factors. Pedestrian factors comprise the trajectory and path selection, the physiological factor, and the psychological factor of the crowd. Equipment factors mainly demonstrate the effect of items imposed by items, equipment, and facilities on crowd density, crowd movement, and crowd evacuation. Environment factors, including natural environment and natural disasters, play a role in crowd movement. Management factors involve the subjectivity of managers, guidance mechanisms, and on-site control on crowd evacuation. Given an underpinning of the theory of risk ergonomics accident causality analysis [28], all risk factors in stampedes are in transitive relations, explaining that the chain development of accident causality ultimately constitutes the accident causality. The schematic relationship is shown in Fig. 1.

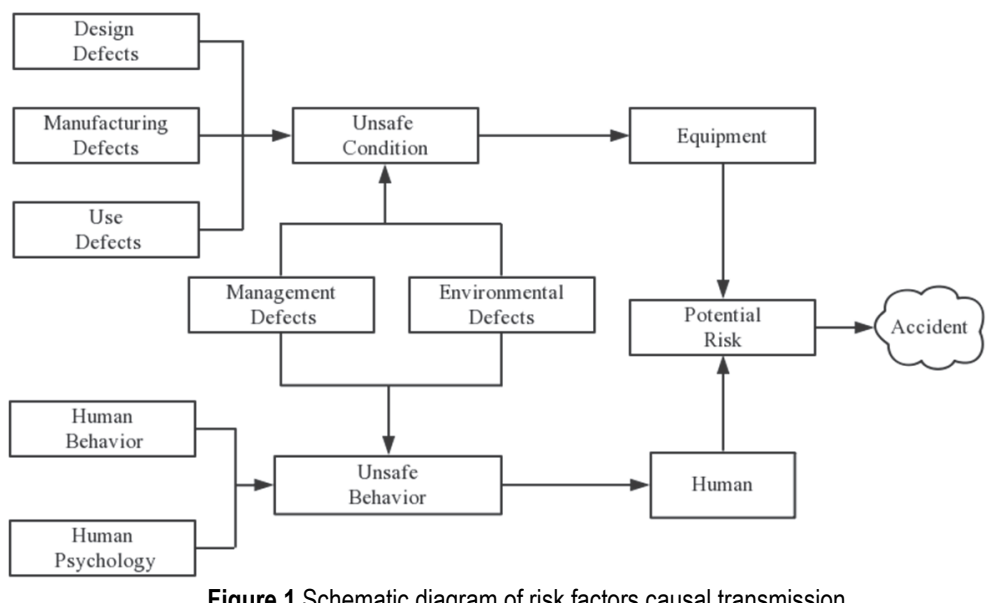

Figure 1 Schematic diagram of risk factors causal transmission 
In accordance with the "pedestrian-equipmentenvironment-management" analysis framework, this study conducts conceptual coding of risk factors by investigating a host of typical cases of the past, which can conspicuously reveal and summarize conceptual factors that trigger and evolve crowd trampling accidents. The analysis process is shown in Tab. 1.

The influencing factors of stampede have been collected and identified through a myriad of case analyses, in which the triggering factors are conceptualized and screened out in a questionnaire method.

Table 1 Conceptual coding analysis of influencing factors of stampede

\begin{tabular}{|c|c|c|}
\hline Stampede & Incident triggering and evolution process & Conceptual coding \\
\hline \multirow{6}{*}{2014 Shanghai Stampede } & Citizens gathered at the Bund to celebrate the New Year, causing 310,000 deaths & Over-density of crowds \\
\hline & $\begin{array}{l}\text { Up and down crowds forged into stalemate in the middle of stairs and formed a } \\
\text { "surge" }\end{array}$ & Direction chaos of crowds \\
\hline & $\begin{array}{c}\text { The suddenly increasing downward pressure on the crowds caused pedestrian } \\
\text { imbalance at the bottom of the stairs }\end{array}$ & Rugged surfaces or steep slope \\
\hline & Pedestrian falling caused by flow imbalance & Pedestrian swarming and falling \\
\hline & $\begin{array}{l}\text { The rescued police ordered the crowds on the stairs back, but they continued to } \\
\text { losing control }\end{array}$ & Insufficient transactions \\
\hline & Panic resulted in crowds falling and trampling & Crowd panic \\
\hline \multirow{4}{*}{$\begin{array}{c}2018 \text { Crowd Stampede at } \\
\text { Bihasariv Train Station in } \\
\text { Bihar, India }\end{array}$} & A large number of students slept on the narrow platform waiting for the train & Over-density of crowds \\
\hline & Near midnight, in a dark environment & Weather condition \\
\hline & $\begin{array}{l}\text { Asbestos tiles dropped at a station, and students mistakenly thought an earthquake } \\
\text { occurred }\end{array}$ & Emergency \\
\hline & The students fled away in a panic & Crowd panic \\
\hline$\ldots$ & $\ldots$ & $\ldots$. \\
\hline
\end{tabular}

\subsection{Centrality Analysis of Influencing Factors Based on SNA Methodology}

SNA is defined to quantitatively analyze the relationship between nodes in the social network structure and exposes the overall structure and individual attributes of the social network erected by the nodes by using matrix and graph theory $[29,30]$. In SNA, "centrality" serves as a standard to measure the superiority and privilege of various index factors in a social structure network [31]. Thus, this study processes quantified data of a crowd stampede system with the application of SNA and then provides a fundamental basis for decision-making of crowd risk management and control on the basis of the SNA results.

Degree centrality represents the number of nodes in the network that are connected to this node. The centrality indicator arguably includes degree and intermediate centrality, the first of which is divided into two types: absolute and $N_{r m}$ centrality. The former refers to the number of nodes directly linked to the point, and the latter means the ratio of the absolute degree centrality to the maximum possible degree of nodes in the graph [32]. Judging from the analysis of stampede risk factors, the greater the " $N_{r m}$ degree centrality" the more likely this factor is to be combined with other factors to cause an incident and be situated in the center of the network, compounded by the higher risk. Intermediate centrality is the sum of the probabilities that a node is on the shortcut of two nodes on the network, implying the node's ability to control the interaction between other nodes. In other words, other factors are positively related to the changes in degree centrality. The specific modelling steps are as follows.

\subsubsection{Establishment of Weighted Influencing Matrix}

The weighted influencing matrix intends to determine the direct influencing degree and influencing matrix between factors. The Delphi method is adopted to identify the influencing intensity between the factors, which is compared and arranged in ascending order of intensity from 0 to 5 . Here, " 0 " represents no relationship, "1" means a relatively weak relationship, "2" indicates a weak relationship, "3" implies a modest relationship, "4" shows a relatively strong relationship, and "5" describes the strongest relationship. Experts in crowd management and control and evacuation industries judge whether a relationship and corresponding influencing degree of those related factors is present. The direct impact matrix $\boldsymbol{X}=\left(x_{i j}\right)_{n \times n}$ is obtained, in which $x_{i j}$ explains the direct influencing degree of the factor $c_{i}$ on the factor $c_{j}$, and $x_{i j}=$ 0 is secured when $i=j$.

\subsubsection{Matrix Symmetry and Binarization}

In a mutual evaluation of the crowd stampede system, due to the interaction and influence evaluation between two related factors based on the subjective cognition of several experts, the mutual scores in the multi-valued matrix may be different. In this study, UCINET software is applied to perform symmetry and binarization processing on the multi-value matrix.

\subsubsection{Indicator Centrality Calculation}

The absolute centrality of the risk factor is the number of other nodes directly connected to the node, registering in $C_{A D}$.

The $N_{r m}$ centrality of the risk factor is the ratio of the absolute centrality of the node to the maximum possible degree among the network nodes, which is denoted by $C_{A D}$ '. The calculation formula is:

$$
C_{A D}{ }^{\prime}=\frac{C_{A D}}{n-1}
$$

In the formula, $n$ is the network scale, namely, the number of risk factors in the network.

The betweenness centrality of the indicator is the sum of the probabilities that a node is on the shortcut of two nodes on the network, which manifests the risk factor's 
ability to control the interaction between other nodes. The formula is:

$$
C_{A B i}=\sum_{j}^{n} \sum_{k}^{n} b_{j k}(i)=\sum_{j}^{n} \sum_{k}^{n} \frac{g_{j k}(i)}{g_{j k}}, j \neq k \neq I, j \prec k
$$

In the formula, $b_{j k}(i)$ represents the ability of node $i$ to control the interaction between node $j$ and node $k . g_{j k}(i)$ is the number of shortcuts between node $j$ and node $k$ passing through node $i . g_{j k}$ is the total number of shortcuts between node $j$ and node $k$. $I$ means the unit matrix.

\subsection{Weight Analysis of Influencing Factors Based on Stampede Accident Statistics}

The weight coefficient refers to the frequency and importance of the influencing factors, with the function of intuitively and systematically describing the intensity of stampedes that is affected by indicators. The coefficient then reveals the incident mechanism. In accordance with the analysis results of influencing factors and conceptual coding, this study adopts mathematical statistical analysis methods to determine the frequency of occurrence of various risk factors in historical cases of stampede worldwide. If $\omega^{(i)}$ is the weighting coefficient of crowd stampede accidents, then $\omega^{(i)}$ represents the contribution rate of the influencing factor to crowd stampedes. The calculation formula of single factor weight is:

$\omega(j)^{(i)}=\frac{j^{(i)}}{n}$

In the formula, $j^{(i)}$ is the frequency of influencing factor $j, i$ is the level of the indicator factor, and $n$ is the total frequency of all influencing factors.

Based on the calculation of the weighting results of risk factors and combined with SNA centrality analysis, the trigger mechanism of crowd stampede accidents is fully revealed from the dimensions of accident network structure, risk factor causality, and indicator effectiveness.

\section{RESUILT ANALYSIS AND DISCUSSION 4.1 Collection of Influencing Factors}

In collaboration with the previous extraction method for the influencing factors of crowd stampede accidents, we collated and sorted out 78 crowd stampedes in the world from 2010 to 2019 through information reported by mainstream media at home and abroad. We then screened out the typical and representative cases with tremendous social effect and causing serious casualties to carry out conceptual coding of influencing factors. On this basis, we conducted interviews with 20 experts on intensive crowd management and control via media such as email, telephone, and video conference. We optimized the selection of influencing factors. On the basis of the collation and analysis of the interview results, and the division framework according to the indicators of "pedestrian-equipment-environment-management", the concise 17 influencing factors of stampede were obtained, as shown in Fig. 2.

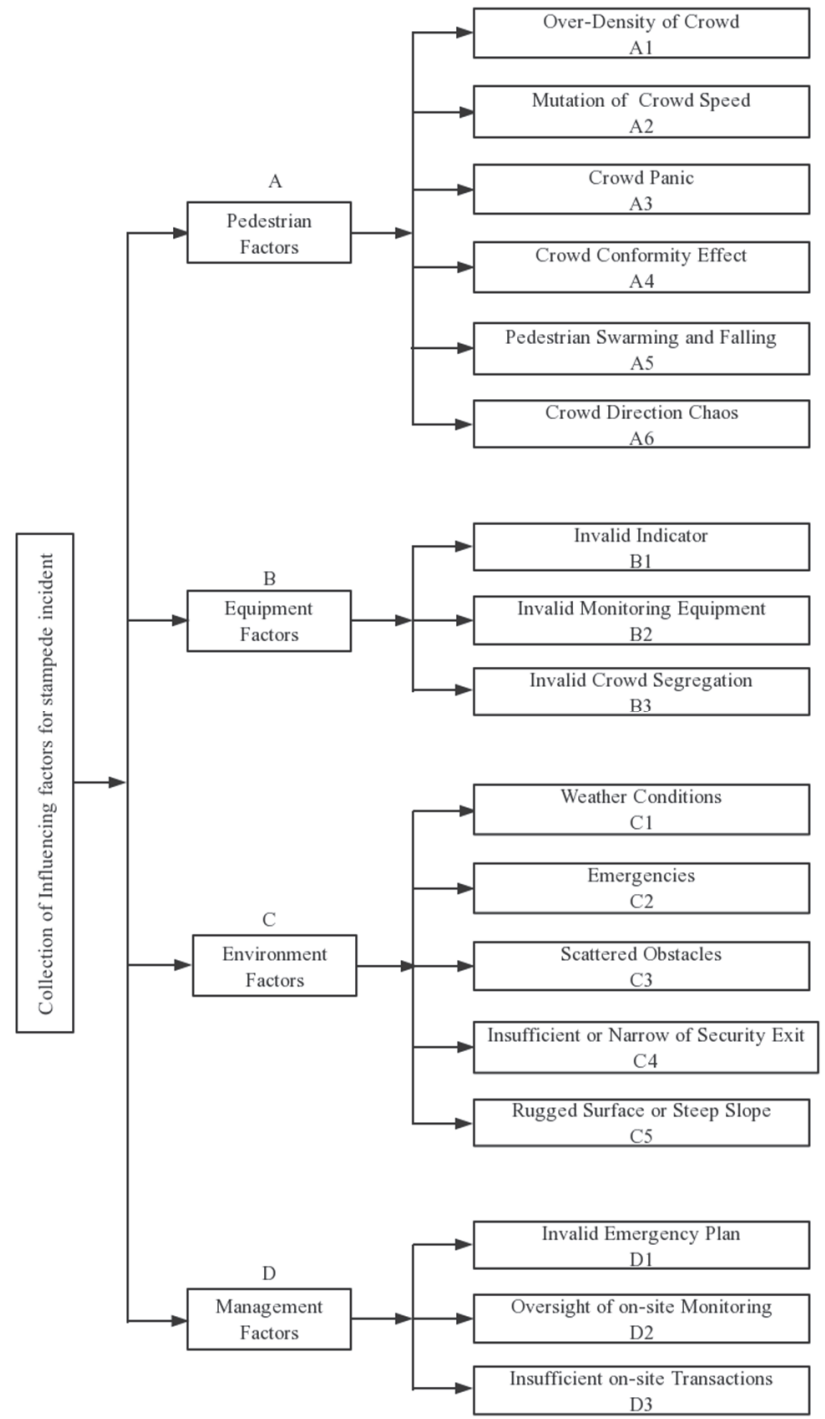

Figure 2 Influencing factor collections of stampedes

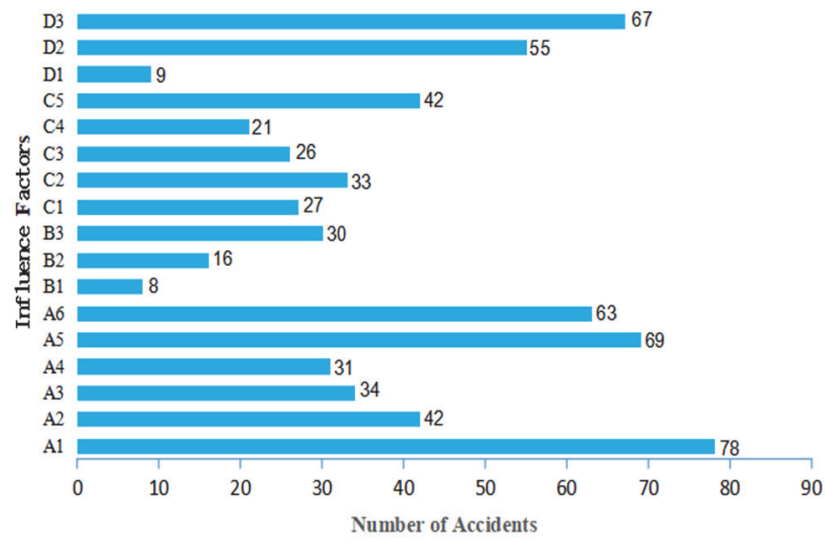

Figure 3 Statistical results of accidents involving different influencing factors

The conceptual coding of influencing factors naturally combs out the time, place, triggering factors, main participants, and casualties and then collects the influencing factors in statistics of the crowd stampede accidents. Considering that an accident is caused by more than one single factor, the total number of influencing factors is greater than 78 . The statistical results are shown in Fig. 3. 


\subsection{Influencing Factor Centrality}

Influencing factors of stampede constitute a complex system, which includes causal feedback between the factors. The relationship between factors is the fundamental condition for SNA analysis. In this study, the Delphi method is used to collect opinions from multiple experts to determine the influence relationship between various factors. At the same time, further refining the relationship intensity between the influencing factors, this study conducts a questionnaire survey on dense crowd research scholars from institutions such as Tsinghua University, China University of Science and Technology, and Wuhan University of Technology, by distributing 40 questionnaires with 36 valid results recovered. Through comprehensive analysis of expert opinions, this study quantifies the relationship intensity between various factors in the social network of crowd stampede accidents, establishing the direct influence matrix of the factor set, as shown in Tab. 2.

Table 2 Multi-value matrix scoring results of various risk factors of the crowd stampede system

\begin{tabular}{|c|c|c|c|c|c|c|c|c|c|c|c|c|c|c|c|c|c|}
\hline$X$ & A1 & A2 & A3 & A4 & A5 & A6 & B1 & B2 & B3 & C1 & $\mathrm{C} 2$ & C3 & $\mathrm{C} 4$ & C5 & D1 & D2 & D3 \\
\hline A1 & 0 & 1 & 2 & 2 & 4 & 2 & 0 & 0 & 3 & 0 & 0 & 0 & 0 & 0 & 0 & 0 & 3 \\
\hline A2 & 3 & 0 & 2 & 3 & 3 & 3 & 0 & 0 & 1 & 0 & 0 & 0 & 0 & 0 & 0 & 0 & 0 \\
\hline A3 & 3 & 4 & 0 & 4 & 4 & 4 & 0 & 0 & 4 & 0 & 0 & 0 & 0 & 0 & 0 & 0 & 3 \\
\hline A4 & 4 & 2 & 1 & 0 & 3 & 1 & 0 & 0 & 1 & 0 & 0 & 0 & 0 & 0 & 0 & 0 & 2 \\
\hline A5 & 4 & 5 & 3 & 3 & 0 & 4 & 0 & 0 & 2 & 0 & 0 & 0 & 0 & 0 & 0 & 0 & 2 \\
\hline A6 & 2 & 1 & 0 & 0 & 3 & 0 & 0 & 0 & 1 & 0 & 0 & 0 & 0 & 0 & 0 & 0 & 4 \\
\hline B1 & 1 & 0 & 0 & 0 & 0 & 1 & 0 & 0 & 0 & 0 & 0 & 0 & 0 & 0 & 0 & 0 & 0 \\
\hline B2 & 2 & 0 & 0 & 0 & 1 & 2 & 0 & 0 & 0 & 0 & 0 & 0 & 0 & 0 & 0 & 4 & 4 \\
\hline B3 & 2 & 2 & 0 & 2 & 2 & 3 & 0 & 0 & 0 & 0 & 0 & 0 & 0 & 0 & 0 & 0 & 3 \\
\hline $\mathrm{C} 1$ & 3 & 1 & 2 & 3 & 2 & 2 & 1 & 1 & 0 & 0 & 0 & 0 & 0 & 0 & 0 & 3 & 3 \\
\hline C2 & 5 & 5 & 5 & 4 & 4 & 3 & 0 & 2 & 3 & 0 & 0 & 0 & 0 & 0 & 0 & 0 & 5 \\
\hline C3 & 2 & 1 & 0 & 1 & 2 & 1 & 0 & 0 & 0 & 0 & 0 & 0 & 2 & 0 & 0 & 2 & 2 \\
\hline C4 & 4 & 3 & 0 & 2 & 3 & 0 & 0 & 0 & 0 & 0 & 0 & 2 & 0 & 0 & 0 & 0 & 1 \\
\hline C5 & 3 & 2 & 0 & 0 & 2 & 0 & 0 & 0 & 0 & 0 & 0 & 0 & 0 & 0 & 0 & 0 & 1 \\
\hline D1 & 3 & 0 & 0 & 0 & 2 & 4 & 0 & 0 & 0 & 0 & 0 & 0 & 0 & 0 & 0 & 0 & 4 \\
\hline D2 & 3 & 1 & 1 & 0 & 2 & 3 & 4 & 0 & 2 & 0 & 3 & 1 & 0 & 1 & 3 & 0 & 5 \\
\hline D3 & 4 & 3 & 4 & 2 & 5 & 5 & 0 & 0 & 4 & 0 & 3 & 0 & 2 & 0 & 4 & 0 & 0 \\
\hline
\end{tabular}

Table 3 Calculation result of centrality of each factor of the crowd stampede system

\begin{tabular}{|c|c|c|c|c|}
\hline Influence Factors & Degree & $N_{r m}$ Degree & Share \\
\hline A1 & 16 & 100 & 0.0930 \\
\hline A2 & 13 & 81.25 & 0.0756 \\
\hline A3 & 10 & 62.5 & 0.05814 \\
\hline A4 & 11 & 68.75 & 0.06395 \\
\hline A5 & 15 & 93.75 & 0.08721 \\
\hline A6 & 14 & 87.5 & 0.08139 \\
\hline B1 & 4 & 25 & 0.02326 \\
\hline B2 & 7 & 43.75 & 0.04070 \\
\hline C1 & 9 & 56.25 & 0.05233 \\
\hline C2 & 10 & 62.5 & 0.05814 \\
\hline C3 & 10 & 62.5 & 0.05814 \\
\hline C5 & 8 & 50 & 0.04651 \\
\hline D1 & 6 & 37.5 & 0.03262 \\
\hline D2 & 5 & 31.25 & 0.03488 \\
\hline D3 & 5 & 31.25 & 0.02907 \\
\hline
\end{tabular}

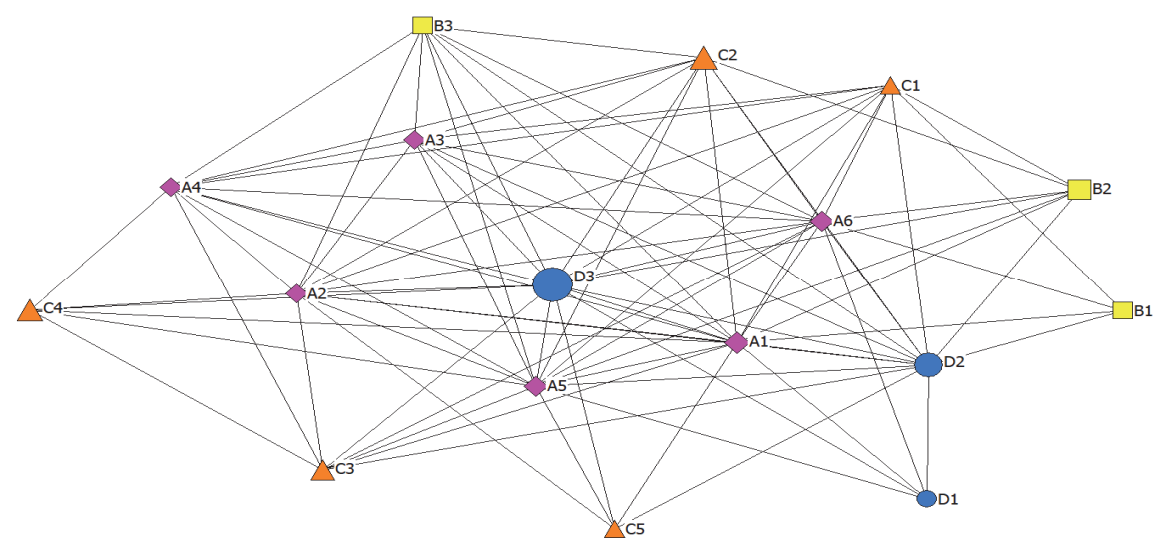

Figure 4 Schematic diagram of centrality analysis of various factors from the SNA perspective

UCINET software is adopted to calculate the degree and intermediate centrality of each risk factor, namely, absolute degree centrality, $N_{r m}$ degree centrality, share, and betweenness. The results are shown in Tab. 3, and the 
factor intermediate centrality relationship is shown in Fig. 4.

$N_{r m}$ degree centrality characterizes the shares of risk factors in a crowd stampede accident network, namely, the centrality and importance of factors in accidents. The greater the $N_{r m}$ degree centrality, the more easily this factor combines with other factors to cause an accident, and the greater the risk. According to the results of centrality analysis of the influencing factors in Tab. 3, the higher risk factors of $N_{r m}$ degree centrality are over-density of crowd (A1), insufficient transactions (D3), pedestrian swarming and falling (A5), crowd direction chaos (A6), oversight of on-site monitoring (D2), mutation of crowd speed (A2), crowd conformity effect (A4), emergencies (C2), weather conditions (C1) and crowd panic (A3). Among them, the over-density of crowds (A1), insufficient on-site transactions (D3), and pedestrian swarming and falling (A5) account for the highest shares, indicating that the three indexes are most likely to merge into other factors to cause incidents. Among the above three factors, insufficient on-site transactions (D3) of the management side is an indirect factor that leads to over-density of crowds (A1). The coupling effect of excessive crowd density and other risk factors triggers pedestrian falling (A5) of swarming crowds, eventually accelerating stampede occurrence.

Intermediate centrality involves the control ability of risk factors in a crowd stampede accident network. In other words, it is the influence of factors on other related risk factors, which is the reason for the increased risk of related factors. In general, the greater the centrality, the more likely this factor will affect other factors and result in stampedes. With the reference of the results of the centrality of the influencing factors in Tab. 3, the risk factors with higher intermediate centrality are as follows: insufficient on-site transactions (D3), oversight of on-site monitoring (D2), emergencies (C2), scattered obstacles (C3), over-density of crowds (A1), and crowd direction chaos (A6). Among them, the greatest weight of insufficient on-site transactions (D3) implies that this sort of risk factor is predisposed to impose an effect on other factors and exert strong control influence. Fig. 4 shows that an insufficient on-site transaction (D3), at the core of the entire network, is closely related to multiple factors. Furthermore, oversight of on-site monitoring (D2), which plays the same management role as D3 does, also has a certain control effect on correlated factors, meaning that management takes the main responsibility for accidents.

\subsection{Factor Weight}

Based on the risk factors of crowd stampede in Fig. 2 and the statistical results in Fig. 3, this study calculates the original data for accessing the weight results of each influencing factor in terms of Formula (1) by using MATLAB software, as shown in Tab. 4.

In Tab. 4, from the perspective of the first-level indicator classification, the most crucial factor leading to trampling accidents is the inherent factor of crowd risk (A), accounting for $48.49 \%$ for the incidence of stampede accidents. This factor is followed by the environmental risk factor of crowds (C), the management factor (D), and the equipment risk factor $(\mathrm{C})$. On the basis of the analysis results of the SNA intermediate centrality, the main influence of the internal risk factor $(\mathrm{A})$ of the crowds is the management factor (D). This finding shows that the safety management agencies, public security departments, and the government have weak links in the investigation and handling of crowd supervision issues, not playing the role of supervision and correction.

Table 4 Weight of risk factors for crowd stampedes
\begin{tabular}{|c|c|c|c|}
\hline $\begin{array}{c}\text { Influence } \\
\text { Factors }\end{array}$ & Frequency & Weight & $\begin{array}{c}\text { Primary Index } \\
\text { Weight }\end{array}$ \\
\hline A1 & 78 & 0.1198 & \multirow{2}{*}{0.4849} \\
\hline A2 & 42 & 0.0645 & \multirow{2}{*}{0.0 .0522} \\
\hline A3 & 34 & 0.0529 \\
\hline A4 & 31 & 0.0476 & \multirow{2}{*}{0.082} \\
\hline A5 & 69 & 0.1060 & \\
\hline A6 & 63 & 0.0968 & \\
\hline B1 & 8 & 0.0123 & \multirow{2}{*}{0.2289} \\
\hline B2 & 16 & 0.0246 & \\
\hline B3 & 30 & 0.0461 & \\
\hline C1 & 27 & 0.0415 & \\
\hline C2 & 33 & 0.0507 & \multirow{2}{*}{0.2013} \\
\hline C3 & 26 & 0.0399 & \\
\hline C4 & 21 & 0.0323 & \\
\hline C5 & 42 & 0.0645 & \\
\hline D1 & 9 & 0.0138 & \\
\hline D2 & 55 & 0.0845 & \\
\hline D3 & 67 & 0.1029 & \\
\hline & & & \\
\hline
\end{tabular}

In the analysis of the secondary indicators, overdensity of crowds (A1), pedestrian swarming and falling (A5), insufficient on-site transactions (D3), crowd direction chaos (A6), and oversight of on-site monitoring (D2) enjoy the largest weight, significantly participating in the stampede. The weighted judgment analysis result is consistent with the SNA $N_{r m}$ degree centrality analysis result. Failure in supervision and security management of dense crowds, such as formulating effective and feasible control measures for crowds and site path planning, easily causes pedestrians to fall and large stampedes worsen. Security management agencies, public security departments, and governments should further strengthen law enforcement in crowd supervision.

\section{CONCLUSIONS}

Aiming at the influencing factors and mechanism of stampede, an SNA method was used to analyze the interaction intensity among factors, a conceptual coding influencing factor set was constructed based on mathematical statistics, and the centrality and weight of influencing factors were determined. Conclusions are as follows.

(1) The stampede mechanism is an intricate system with multiple coupled factors. The SNA analysis method can better integrate the "micro" network of indicators with the "macro" structure of the disaster-causing system through factor node relationships, fully consider the correlation mode and effect of accident risk factors, and reveal the occurrence mechanism of accidents from the perspective of network analysis.

(2) The weight analysis results based on the SNA analysis model combined with historical data can effectively determine the main risk factors of trampling incidents. Factors such as excessive crowd density, poor on-site transactions, pedestrian crowding and falling, 
crowd direction chaos, and oversight of on-site supervision are likely to cause accidents in combination with other factors. The results of degree centrality and weight analysis provide directional guidance for the control measures of stampede accidents.

(3) The SNA model's intermediate centrality analysis can accurately divide the impact level and risk transmission path of risk factors. Ineffective on-site transactions, oversight of on-site monitoring, emergencies, and scattered obstacles have strong control and influence over other related risk factors, deteriorating other related risk factors. The analysis fully reveals the importance and existing problems of security management agencies in the supervision of swarming crowds.

In conclusion, the SNA method is used to analyze the risk factors of crowd stampede accidents. The method can determine the impact level and mechanism of risk factors and can control the risk of pressing crowds. The method provides a simple and reliable analysis model for distinguishing the importance of risk factors, impact levels, and influencing paths in social public security management.

\section{Acknowledgements}

This study was supported by the Science and technology planning project of Henan Province in China (202102310624, 192102310487).

\section{REFERENCES}

[1] Bork, T., Etzold, B., Gransow, B., et al. (2016). Agency and the making of transient urban spaces: Examples of migrants in the city in the Pearl River Delta, China, and Dhaka, Bangladesh. Population, Space and Place, 22(2), 128-145. https://doi.org/10.1002/psp.1890

[2] Suryani, E., Hendrawan, R. A., Adipraja, P. F. E., et al. (2020). System dynamics simulation model for urban transportation planning: A case study. International Journal of Simulation Modelling, 19(1), 5-16. https://doi.org/10.2507/IJSIMM19-1-493

[3] Zhou, M., Dong, H., Zhao, Y., et al. (2019). Optimization of crowd evacuation with leaders in urban rail transit stations. IEEE Transactions on Intelligent Transportation Systems, 20(12), 4476-4487. https://doi.org/10.1109/TITS.2018.2886415

[4] Zhang, Z., Zhang, Z., \& Yang, J. F. (2018). Risk factors of secondary accidents based on the Bayesian hierarchical model. Journal of Engineering Science \& Technology Review, 11(5), 53-60. https://doi.org/10.25103/jestr.115.07

[5] Zhang, Y. L. \& Zhou, X. B. (2017). The occurrence laws of campus stampede accidents in China and its prevention and control measures. Natural Hazards, 87(2), 659-673. https://doi.org/10.1007/s11069-017-2785-7

[6] Mao, Y., Yang, S., Li, Z., et al. (2020). Personality trait and group emotion contagion based crowd simulation for emergency evacuation. Multimedia Tools \& Applications, 79, 3077-3104. https://doi.org/10.1007/s11042-018-6069-3

[7] Mccaffrey, S., Wilson, R., \& Konar, A. (2017). Should I stay or should I go now? Or should I wait and see? Influences on wildfire evacuation decisions. Risk Analysis, 38(7), 13901404. https://doi.org/10.1111/risa.12944

[8] Zong, X., Wang, C., Du, J., et al. (2019). Tree hierarchical directed evacuation network model based on artificial fish swarm algorithm. International Journal of Modern Physics C. 30(11), 1117-1140. https://doi.org/10.1142/S0129183119500979
[9] Maureen, P., Mary, R., Craig, T., et al. (2014). Wildfire evacuation scenario in Colorado: comparison of adapted four-step metropolitan planning organization modelling results and planning process findings with actual experience. Transportation Research Record, 2430(01), 133-144. https://doi.org/10.3141/2430-14

[10] Ding, Y., Weng, F., \& Yang, L. (2019). Study about influence of doors' opening degree on crowds' evacuation based on simulation. International Journal of Modern Physics C, 30(11), 1950096. https://doi.org/10.1142/S0129183119500967

[11] Arumugam, G. P., Augustine, J., Golin, M. J., et al. (2019). Minmax regret k-sink location on a dynamic path network with uniform capacities. Algorithmica, 81(9), 3534-3585. https://doi.org/10.1007/s00453-019-00589-2

[12] Zeng, L., Gao, J., Wang, Q., et al. (2018). A risk assessment approach for evaluating the impact of toxic contaminants released indoors by considering various emergency ventilation and evacuation strategies. Risk analysis, 38(11), 2379-2399. https://doi.org/10.1111/risa.13132

[13] Vinayak, D., Thomas, M., Brian, W., et al. (2011). Validation techniques for region-level microscopic mass evacuation traffic simulations. Transportation Research Record, 2229(01), 66-74. https://doi.org/10.3141/2229-08

[14] Collins, A. J., Michael, Robinson R., Jordan, C., et al. (2017). Development of a traffic incident model involving multiple municipalities for inclusion in large microscopic evacuation simulations. International Journal of Disaster Risk Reduction, 31, 1223-1230. https://doi.org/10.1016/j.jidrr.2017.12.010

[15] Wang, Z., Liu, M., \& Zhao, Y. (2018). Analysis of trample disaster and a case study-Mihong bridge fatality in China in 2004. Safety Science, 46(08), 1255-1270. https://doi.org/10.1016/j.ssci.2007.08.002

[16] Yan, L., Wang, T., Hui, D., et al. (2013). Research on risk assessment DEA model of crowd crushing and trampling accidents in subway stations. China Safety Science Journal, 23(10), 100-104.

[17] Illiyas, F. T., Mani, S. K., Pradeepkumar, A. P., et al. (2013). Human stampedes during religious festivals: A comparative review of mass gathering emergencies in India. International Journal of Disaster Risk Reduction, 5(9), 10-18. https://doi.org/10.1016/j.jidrr.2013.09.003

[18] Alaska, Y. A., Aldawas, A. D., Aljerian, N. A., et al. (2017). The impact of crowd control measures on the occurrence of stampedes during Mass Gatherings: The Hajj experience. Travel Medicine \& Infectious Disease, 15, 67-70. https://doi.org/10.1016/j.tmaid.2016.09.002

[19] Yu, F., Song, Y., Huo, F., et al. (2016). Mechanism and risk evaluation of pedestrian stampede accidents in public places of cities-A level influence model based on the event system theory (EST). Science Research Management, 37(12), 162169.

[20] Liu, Z., Liu, T., Ma, M., et al. (2018). A perception-based emotion contagion model in crowd emergent evacuation simulation. Computer Animation \& Virtual Worlds, 29(04), 1-14. https://doi.org/10.1002/cav.1817

[21] Liu, S. S., Liu, J., \& Wei, W. (2019). Simulation of crowd evacuation behavior in outdoor public places: A model based on Shanghai stampede. International Journal of Simulation Modelling, 18(1), 86-99. https://doi.org/10.2507/IJSIMM18(1)464

[22] Helbing, D. \& Mukerji, P. (2012) Crowd disasters as systemic failures: analysis of the Love Parade disaster. EPJ Data Science, 1(7), 1-40. https://doi.org/10.1140/epjds7

[23] Ramesh, M. V., Shanmughan, A., \& Prabha, R. (2014). Context aware ad hoc network for mitigation of crowd disasters. Ad Hoc Networks, 18(3), 55-70. https://doi.org/10.1016/j.adhoc.2013.02.006 
[24] Shiwakoti, N., Tay, R., Stasinopoulos, P., et al. (2017). Likely behaviours of passengers under emergency evacuation in train station. Safety Science, 91, 40-48. https://doi.org/10.1016/j.ssci.2016.07.017

[25] Zhao, Y., Li, M., Lu, X., et al. (2017). Optimal layout design of obstacles for panic evacuation using differential evolution. Physica A Statistical Mechanics \& Its Applications, 465, 175-194. https://doi.org/10.1016/j.physa.2016.08.021

[26] Dias, C., Sarvi, M., Shiwakoti, N., et al. (2013). Investigating collective escape behaviours in complex situations. Safety Science, 60(60), 87-94 https://doi.org/10.1016/j.ssci.2013.07.005

[27] He, W., Zhang, Z., Lu, L., et al. (2018). Analysis on the influence factors of accident severity: evidence from urban rivercrossing tunnels in Shanghai of China. Journal of Engineering Science \& Technology Review, 11(5):100-108. https://doi.org/10.25103/jestr.115.12

[28] Elvik, R. (2011). Assessing causality in multivariate accident models. Accident Analysis \& Prevention, 43(1), 3-264. https://doi.org/10.1016/j.aap.2010.08.018

[29] Shafiq, O., Alhajj, R., \& Rokne, J. G. (2015). On personalizing Web search using social network analysis. Information Sciences, 314, 55-76. https://doi.org/10.1016/j.ins.2015.02.029

[30] Zheng, X., Le, Y., Chan, A. P., Hu, Y., \& Li, Y. (2016). Review of the application of social network analysis (SNA) in construction project management research. International Journal of Project Management, 34(7), 1214-1225. https://doi.org/10.1016/j.jproman.2016.06.005

[31] Park, J. (2011). The use of a social network analysis technique to investigate the characteristics of crew communications in nuclear power plants-A feasibility study. Reliability Engineering \& System Safety, 96(10), 1275-1291. https://doi.org/10.1016/j.ress.2011.05.003

[32] Medina, Richard, M. (2014). Social Network Analysis: A case study of the Islamist terrorist network. Security Journal, 27(1), 97-121. https://doi.org/10.1057/sj.2012.21

\section{Contact information:}

Xinyao GUO, PhD, Lecturer

(Corresponding author)

School of Civil Aviation,

Zhengzhou University of Aeronautics,

Zhengzhou, Henan, China

Email: sggxy@126.com

Linqing NIU, PhD, Lecturer

School of Civil Aviation,

Zhengzhou University of Aeronautics,

Zhengzhou, Henan, China

E-mail: niulinqing@sina.com

Hongyan GUAN, PhD, Lecturer

School of Civil Aviation,

Zhengzhou University of Aeronautics,

Zhengzhou, Henan, China

E-mail: guanhongyan321@163.com 